\title{
Reducing Computational Costs in the Basic Perturbation Lemma ${ }^{\star}$
}

\author{
Ainhoa Berciano ${ }^{1}$, María José Jiménez ${ }^{2}$, and Pedro Real ${ }^{2}$ \\ ${ }^{1}$ Dpto. Matemática Aplicada, Estadística e Investigación Operativa, \\ Universidad del País Vasco, Barrio Sarriena s/n, 48940 Leioa (Vizcaya), Spain \\ ainhoa.berciano@ehu.es \\ ${ }^{2}$ Dpto. de Matemática Aplicada I, Universidad de Sevilla, \\ Avda. Reina Mercedes s/n, 41012 Sevilla, Spain \\ \{majiro, real\}@us.es
}

\begin{abstract}
Homological Perturbation Theory [11,13, is a well-known general method for computing homology, but its main algorithm, the $B a$ sic Perturbation Lemma, presents, in general, high computational costs. In this paper, we propose a general strategy in order to reduce the complexity in some important formulas (those following a specific pattern) obtained by this algorithm. Then, we show two examples of application of this methodology.
\end{abstract}

\section{Introduction}

Most algorithms in Algebraic Topology and Homological Algebra carry high computational costs. We are concerned with the search of techniques that cut down complexity in processes from these areas. In order to compute homology, two important tools in Homological Algebra are, on the one hand, the notion of contraction [6, a special type of homotopy equivalence, between differential graded modules (DG-modules) and, on the other hand, the Basic Perturbation Lemma (BPL), which allows one to generate a new contraction by "perturbation" of a previous one (see 22 or 18]). In fact, the BPL, is an algorithm whose input is a contraction between two differential graded modules together with a "perturbation" of the differential structure on the first DG-module; the output is a contraction from the perturbed module onto the second module, whose differential comes out to be modified. The formulas of the latter contraction as well as the modification in the differential structure of the second DG-module often imply high computational costs. Thanks to a tool introduced in this paper, compatible grading, we are able to reduce this complexity in time and space. In fact, we establish some proper conditions under which the calculus implied in the computation of several formulas obtained by the BPL, with a specific structure, can be significantly cut down. However, the improvement achieved in computation will depend on the specific morphisms implied, as well as the compatible grading considered.

^ Partially supported by the PAICYT research project FQM-296 and the UPV-EHU project 00127.310-E-15916. 
We describe two important applications, showing the advantages from the computational point of view in each case. The first one is related to the algebraic structure of the homology of a truncated polynomial algebra. Another example of application of the general strategy presented here is the so-called inversion theory, which was born in [17] and was later used in [4] and [14] in order to simplify the computation of the perturbed differential of a 1-homological model for a commutative DGA-algebra. We must say that we only recall here these results, under the new viewpoint.

This paper is divided as follows. First, we give some preliminaries and notations. Secondly, we establish the theory of compatible gradings, which represents the main original contribution of this paper. Finally, two different applications of this novel strategy for reducing the computational costs of the BPL are shown in the last two sections.

\section{Preliminaries and Notations}

Here we will collect some basic definitions and results in the context of Homological Algebra, as well as the notations that we will adopt. See [5], 16] or [19] for further information.

Take a commutative ground ring with unit, $\Lambda$. We will work with differential graded modules, DG-modules, which are graded modules endowed with a morphism $d$ of graded modules of degree -1 such that $d d=0$. A graded module $M$ is connected whenever $M_{0}=\Lambda$ in which case, the graded module $\bar{M}$ is defined as $\bar{M}_{n}=M_{n}$ for $n>1$ and $\bar{M}_{0}=0$.

We strictly adhere to the Koszul conventions with regard to signs, meaning that if $f: M \rightarrow M^{\prime}$ and $g: N \rightarrow N^{\prime}$ are both DG-module morphisms, then

$$
(f \otimes g)(x \otimes y)=(-1)^{|g||x|} f(x) \otimes g(y) .
$$

Given a DG-module $\left(M, d_{M}\right)$, the suspension of $M$ is the DG-module $(s M$, $\left.d_{s M}\right)$, where $(s M)_{n}=M_{n-1}$ and $d_{s M}=-d_{M}$. Analogously, the desuspension of $M$ is given by $\left(s^{-1} M\right)_{n}=M_{n+1}$ with differential $-d_{M}$. We will denote by $\uparrow$ and $\downarrow$ the suspension and desuspension morphisms which shift the degree by +1 and -1 , respectively.

Given a DG-module $(M, d)$, the tensor module of $M, T(M)$, is constructed as follows:

$$
T(M)=\bigoplus_{n \geq 0} M^{\otimes n} .
$$

The (tensor) grading of a homogeneous element of $T(M), a_{1} \otimes \cdots \otimes a_{n}$ is given by $\sum_{i=1}^{n}\left|a_{i}\right|$. The differential structure in $T(M)$ is provided by the tensor differential,

$$
d_{t}=\sum_{i=0}^{n-1} 1^{\otimes i} \otimes d_{M} \otimes 1^{\otimes n-i-1} .
$$

Any morphism of DG-modules $f: M \rightarrow N$ induces another one $T(f): T(M) \rightarrow$ $T(N)$, being $\left.T(f)\right|_{M \otimes n}=f^{\otimes n}$. 
If a $\mathrm{DG}$-module $A$ is endowed with an associative product with unit, it is called a $D G$-algebra, $\left(A, d_{A}, \mu_{A}\right)$. Sometimes we will use the notation $*_{A}$ for the product on $A$. A $D G$-coalgebra is a $D G$-module provided with a compatible coproduct and counit.

There are several examples of connected commutative DG-algebras with null differential:

- The polynomial DG-algebra $P(v, 2 n)$, generated by $v$ of degree $2 n$, where $n$ is a positive integer. The product is the usual one of monomials i.e., $v^{i} v^{j}=$ $v^{i+j}$.

- The truncated polynomial $D G$-algebra $Q_{p}(v, 2 n)$, which is the quotient algebra $P(v, 2 n) /\left(v^{p}\right)$.

- The exterior DG-algebra $E(u, 2 n+1), n \geq 0$, with algebra generator $u$ of degree $2 n+1$ and trivial product $u^{2}=0$.

- The divided polynomial $D G$-algebra $\Gamma(w, 2 n), n \geq 1$, generated by $\gamma_{1}(w)=w$ $\left(\gamma_{0}(w)=1\right)$ with product the one given by $\gamma_{k}(w) \gamma_{h}(w)=\frac{(k+h) !}{k ! h !} \gamma_{k+h}(w)$;

Given a connected DG-algebra $A$, one can construct the reduced bar construction of $A, \bar{B}(A)$, whose underlying module is $T(s \bar{A})$. A typical element of $\bar{B}(A)$, is denoted by $\bar{a}=\left[a_{1}|\cdots| a_{n}\right] \in(s \bar{A})^{\otimes n}$. The total differential $d_{\bar{B}}$ is given by $d_{\bar{B}}=d_{t}+d_{s}$, being $d_{t}$ the natural one on the tensor module and $d_{s}$ the simplicial differential, that depends on the product on $A$. This DG-module is endowed with a structure of DG-coalgebra by the natural coproduct $\Delta_{\bar{B}}$ defined on the tensor module:

$$
\Delta_{\bar{B}}\left(\left[a_{1}|\cdots| a_{r}\right]\right)=\sum_{i=0}^{r}\left[a_{1}|\cdots| a_{i}\right] \otimes\left[a_{i+1}|\cdots| a_{r}\right] .
$$

If $A$ is a commutative DG-algebra, $\bar{B}(A)$ is endowed with an additional structure of algebra by the shuffle product, $\star$.

Let $p$ and $q$ be two nonnegative integers, a $(p, q)$-shuffle is defined as a permutation $\pi$ of the set $\{0,1, \ldots, p+q-1\}$ such that $\pi(i)<\pi(j)$ whenever $0 \leq i<j \leq p-1$ or $p \leq i<j \leq p+q-1$.

Notice that there are $\left(\begin{array}{c}p+q \\ p\end{array}\right)$ different $(p, q)$-shuffles.

So, the shuffle product $\star$ on $\bar{B}(A)$, is defined (up to sign) by:

$$
\left[a_{1}|\cdots| a_{p}\right] \star\left[b_{1}|\cdots| b_{q}\right]=\sum_{\pi \in\{(p, q)-\text { shuffles }\}} \pm\left[c_{\pi(0)}|\cdots| c_{\pi(p-1)}\left|c_{\pi(p)}\right| \cdots \mid c_{\pi(p+q-1)}\right] ;
$$

where $\left(c_{0}, \ldots, c_{p-1}, c_{p}, \ldots, c_{p+q-1}\right)=\left(a_{1}, \ldots, a_{p}, b_{1}, \ldots b_{q}\right)$.

Given a simply connected DG-coalgebra $C$, the reduced cobar construction, $\bar{\Omega}(C)$, is a DG-algebra whose underlying module is $T\left(s^{-1} \bar{C}\right)$. A typical element of $\bar{\Omega}(C)$ will be written $\bar{c}=\left\langle c_{1}|\cdots| c_{n}\right\rangle$. The total differential $d_{\bar{\Omega}}$ is given by the sum of the tensor differential and the cosimplicial differential:

$$
d_{\text {cos }}=\sum_{i=0}^{n-1} 1^{\otimes i} \otimes \downarrow^{\otimes 2} \Delta_{C} \uparrow \otimes 1^{\otimes n-i-1} .
$$


The product $\mu_{\bar{\Omega}}$ is the natural one on the underlying module, which works by juxtaposition.

Now we briefly recall the main concepts from Homological Perturbation Theory that we will use in this paper.

A contraction $c:\{N, M, f, g, \phi\}[6,13$, also denoted by $(f, g, \phi): N \stackrel{c}{\Rightarrow} M$, from a DG-module $\left(N, d_{N}\right)$ to another one $\left(M, d_{M}\right)$ is a especial type of homotopy equivalence given by the morphisms $f, g$ and $\phi ; f: N_{*} \rightarrow M_{*}$ and $g: M_{*} \rightarrow N_{*}$ are two morphisms of DG-modules and $\phi: N_{*} \rightarrow N_{*+1}$ is a homotopy operator. This way, apart from the conditions

$$
\text { (c1) } f g=1_{M}, \quad(c 2) \phi d_{N}+d_{N} \phi+g f=1_{N},
$$

the following ones must be satisfied

$$
(c 3) f \phi=0, \quad(c 4) \phi g=0, \quad(c 5) \phi \phi=0 .
$$

Given two contractions of DG-modules

$$
c_{i}:\left\{N_{i}, M_{i}, f_{i}, g_{i}, \phi_{i}\right\} \quad i=1,2,
$$

one can construct the following ones [10,11]:

1. The suspension contraction of $c_{1}, s\left(c_{1}\right)$, which consists in taking the suspension DG-modules and the induced morphisms:

$$
s\left(c_{1}\right):\left\{s\left(N_{1}\right), s\left(M_{1}\right), f_{1}, g_{1},-\phi_{1}\right\} .
$$

2. The tensor module contraction, $T\left(c_{1}\right)$, obtained by taking the tensor modules of $M_{1}$ and $N_{1}$ and the induced morphisms,

$$
T\left(c_{1}\right):\left\{T\left(N_{1}\right), T\left(M_{1}\right), T\left(f_{1}\right), T\left(g_{1}\right), T\left(\phi_{1}\right)\right\}
$$

where

$$
\left.T\left(\phi_{1}\right)\right|_{N_{1}^{\otimes n}}=\phi_{1}^{[\otimes n]}=\sum_{i=0}^{n-1} 1^{\otimes i} \otimes \phi_{1} \otimes\left(g_{1} f_{1}\right)^{\otimes n-i-1} .
$$

3. The tensor product contraction:

$$
c_{1} \otimes c_{2}:\left\{N_{1} \otimes N_{2}, M_{1} \otimes M_{2}, f_{1} \otimes f_{2}, g_{1} \otimes g_{2}, \phi_{1} \otimes g_{2} f_{2}+1_{N} \otimes \phi_{2}\right\} .
$$

4. In the case that $N_{2}=M_{1}$, the composition contraction, given by:

$$
c_{2} \circ c_{1}:\left\{N_{1}, M_{2}, f_{2} f_{1}, g_{1} g_{2}, \phi_{1}+g_{1} \phi_{2} f_{1}\right\} .
$$

Let $N$ be a graded module and let $f: N \rightarrow N$ be a morphism of graded modules. The morphism $f$ is called to be pointwise nilpotent if for each $x \in N$, $x \neq 0$, there exists a positive integer $n$ such that $f^{n}(x)=0$. A perturbation of a DG-module $N$ consists in a morphism of graded modules $\delta: N \rightarrow N$ of degree -1 , such that $\left(d_{N}+\delta\right)^{2}=0$. A perturbation datum of the contraction 
$c:\{N, M, f, g, \phi\}$ is a perturbation $\delta$ of the DG-module $N$ such that the composition $\phi \delta$ is pointwise nilpotent.

The key in the Homological Perturbation Theory is the Basic Perturbation Lemma (briefly, BPL) [18, 2, 8, 15, which is an algorithm whose input is a contraction of DG-modules $c:\{N, M, f, g, \phi\}$ and a perturbation datum $\delta$ of $c$ and whose output is a new contraction $c_{\delta}$. The only requirement is the pointwise nilpotency of the composition $\phi \delta$, that guarantees that the sums involved on the formulas are finite for each $x \in N$.

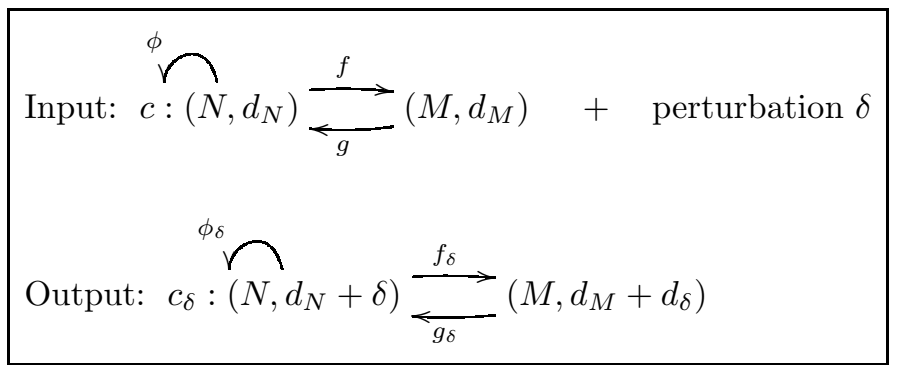

where $f_{\delta}, g_{\delta}, \phi_{\delta}, d_{\delta}$ are given by the formulas

$$
d_{\delta}=f \delta \Sigma_{c}^{\delta} g ; \quad f_{\delta}=f\left(1-\delta \Sigma_{c}^{\delta} \phi\right) ; \quad g_{\delta}=\Sigma_{c}^{\delta} g ; \quad \phi_{\delta}=\Sigma_{c}^{\delta} \phi ;
$$

and $\Sigma_{c}^{\delta}=\sum_{i \geq 0}(-1)^{i}(\phi \delta)^{i}$.

\section{Compatible Gradings}

Let us consider a contraction of DG-modules $c:\{N, M, f, g, \phi\}$ and a perturbation datum for this contraction, $\delta$. The BPL allows one to construct, under certain conditions, a new contraction $c_{\delta}:\left\{\left(N, d_{N}+\delta\right),\left(M, d_{M}+d_{\delta}\right), f_{\delta}, g_{\delta}, \phi_{\delta}\right\}$. The first motivation of this paper was the search of a general way of determining "classes" of elements for which the final result in the calculation of $d_{\delta}$ would be zero. However, the results obtained will mean a possibility of reducing costs in any formula containing the composition $f(\delta \phi)^{i}$. In fact, the pattern

$$
f \delta \phi \delta \phi \cdots \delta \phi \psi
$$

repeatedly appears inside several formulas obtained by BPL (included $d_{\delta}$ ) where $\psi$ can be different compositions of morphisms. Obviously, there is a possibility of reducing the complexity of the morphisms with the pattern above by looking for the elements such that the application of $(\delta \phi)^{i}$ to them belongs to the kernel of $f$. This simple idea is the key of the original theory developed in this section.

Definition 1. Let $c:\{N, M, f, g, \phi\}$ be a contraction of DG-modules. Let $S$ be a submodule of $N$ and $\mathcal{G}=\left\{S_{i}\right\}_{i \geq 0}$ be a grading on $S$ over the nonnegative integers, that is, $S=\bigoplus_{i \geq 0} S_{i}$. This grading is called $c^{-}$-compatible if it satisfies the following conditions: 


$$
\begin{aligned}
& -f\left(S_{i}\right)=0 \text { for } i \geq 1 \\
& -\phi\left(S_{i}\right) \subset \bigoplus_{j \geq i+1} S_{j} .
\end{aligned}
$$

The degree of an element with respect to this new grading will be called $\mathcal{G}$-degree.

Definition 2. Let $c:\{N, M, f, g, \phi\}$ be a contraction of DG-modules, $S \subset N$ a submodule, $\mathcal{G}$ a $c$-compatible grading on $S$ and $\delta$ a perturbation datum for the contraction $c$. The grading $\mathcal{G}$ on $S$ is called to be $(c, \delta)$-compatible if

$$
\delta\left(S_{i}\right) \subset \bigoplus_{j \geq i-1} S_{j}
$$

Taking into account these definitions, it is immediate to state the following proposition.

Proposition 1. Let $c:\{N, M, f, g, \phi\}$ be a contraction of $D G$-modules, $S \subset N$ a submodule, $\delta$ a perturbation datum for $c$ and $\mathcal{G}=\left\{S_{k}\right\}_{k \geq 0} a(c, \delta)$-compatible grading on $S$. Then,

$$
f(\delta \phi)^{i}\left(\bigoplus_{k \geq 1} S_{k}\right)=0 \quad \text { for all } i \geq 0 .
$$

Proof. It is obvious, because of the previous definitions, that the composition $\delta \phi$ keeps the $\mathcal{G}$-degree the same or increases it, that is,

$$
(\delta \phi)^{i}\left(S_{j}\right) \subset \bigoplus_{k \geq j} S_{k}
$$

for any $i$. This way, if $j \geq 1, f(\delta \phi)^{i}\left(S_{j}\right)=0$.

The desired consequence of this result is to find a reduction in complexity of formulas containing the sequence of morphisms $f(\delta \phi)^{i}$ when they are applied to elements of the submodule $S$. For instance, the calculus of the perturbed differential $d_{\delta}$, as well as the projection $f_{\delta}$ obtained from the application of the $\mathrm{BPL}$ to the initial contraction could be reduced: on the one hand, $(\delta \phi)$ will only have to be applied to elements of $\mathcal{G}$-degree zero and, on the other hand, there will only have to consider summands of $\phi$ giving place to elements with $\mathcal{G}$-degree 1 , at the same time that $\delta$ must provide elements of $\mathcal{G}$-degree 0 .

We now wonder what conditions are needed in order to establish compatible gradings for tensor product and composition of contractions. In the case of the tensor product, the compatible gradings have a nice behavior.

Theorem 1. Let $c:\{N, M, f, g, \phi\}$ and $c^{\prime}:\left\{N^{\prime}, M^{\prime}, f^{\prime}, g^{\prime}, \phi^{\prime}\right\}$ be two contractions, let $\mathcal{G}=\left\{S_{k}\right\}_{k \geq 0}$ and $\mathcal{G}^{\prime}=\left\{S_{k}^{\prime}\right\}_{k \geq 0}$ be c-compatible and $c^{\prime}$-compatible gradings of respective submodules $S$ of $N$ and $S^{\prime}$ of $N^{\prime}$. Then the natural grading on the tensor product $\mathcal{G} \otimes \mathcal{G}^{\prime}=\left\{\left(S \otimes S^{\prime}\right)_{k}\right\}_{k \geq 0}$, with $\left(S \otimes S^{\prime}\right)_{k}=\bigoplus_{i+j=k}\left(S_{i} \otimes S_{j}^{\prime}\right)$, is $c \otimes c^{\prime}$-compatible. 
Proof. - Obviously, $\left(f \otimes f^{\prime}\right)\left(S_{i} \otimes S_{j}^{\prime}\right)=0$ if $i+j \geq 1$;

$$
\begin{gathered}
-\left(\phi \otimes g^{\prime} f^{\prime}+1 \otimes \phi^{\prime}\right)\left(S_{i} \otimes S_{j}^{\prime}\right) \subset \bigoplus_{k \geq i+j+1}\left(S \otimes S^{\prime}\right)_{k}, \text { since } \phi\left(S_{i}\right) \subset \bigoplus_{k \geq i+1} S_{k} \text { and } \\
f^{\prime}\left(S_{j}^{\prime}\right)=0 \text { if } j \geq 1 \text { and, on the other hand, } \phi^{\prime}\left(S_{j}^{\prime}\right) \subset \bigoplus_{k \geq j+1} S_{k}^{\prime} .
\end{gathered}
$$

We can extend this result, in a natural way, to the tensor module.

Corollary 1. Let $c:\{N, M, f, g, \phi\}$ be a contraction and $\mathcal{G}=\left\{S_{k}\right\}_{k \geq 0}$ a c-compatible grading. Take the tensor module contraction $T(c)$, then the natural grading on the tensor module $T(S) \subset T(N), T(\mathcal{G})=\left\{(T(S))_{k}\right\}_{k \geq 0}$, with $(T(S))_{k}=\bigoplus_{i_{1}+\cdots+i_{n}=k}\left(S_{i_{1}} \otimes \cdots \otimes S_{i_{n}}\right)$ is $T(c)$-compatible.

The natural grading on the tensor product of two DG-modules is also compatible with the perturbation naturally induced by both perturbations of the initial contractions.

Proposition 2. Under the conditions in the theorem 1 , let $\delta$ and $\delta^{\prime}$ be perturbation data of $c$ and $c^{\prime}$, respectively, such that the gradings $\mathcal{G}$ and $\mathcal{G}^{\prime}$ on $S$ and $S^{\prime}$ are $(c, \delta)$ and $\left(c^{\prime}, \delta^{\prime}\right)$-compatible, respectively. Then, the grading $\mathcal{G} \otimes \mathcal{G}^{\prime}$ on $S \otimes S^{\prime}$ is $\left(c \otimes c^{\prime}, \delta \otimes 1+1 \otimes \delta^{\prime}\right)$-compatible.

Corollary 2. Let $c:\{N, M, f, g, \phi\}$ be a contraction and $\mathcal{G}=\left\{S_{k}\right\}_{k \geq 0}$ a ccompatible grading on $S \subset N$; let $\delta$ be a perturbation datum of c such that the grading $\mathcal{G}$ is $(c, \delta)$-compatible. Then, the grading $T(\mathcal{G})$ on $T(S)$ is $\left(T(c), \delta_{t}\right)$ compatible, where $\left.\delta_{t}\right|_{N \otimes n}=\sum_{i=0}^{n-1} 1^{\otimes i} \otimes \delta \otimes 1^{\otimes n-i-1}$.

Concerning the composition of contractions, we are able to state some conditions under which it is possible to establish a compatible grading for a contraction $c^{\prime} \circ c$, starting from a contraction $c^{\prime}$ with a compatible grading.

Theorem 2. Let $c:\{N, M, f, g, \phi\}$ and $c^{\prime}:\left\{M, N^{\prime}, f^{\prime}, g^{\prime}, \phi^{\prime}\right\}$ be two contractions, let $\mathcal{G}^{\prime}=\left\{M_{k}\right\}_{k \geq 0}$ be a $c^{\prime}$-compatible grading on a submodule of $M$. Take a grading $\mathcal{G}=\left\{N_{k}\right\}_{k \geq 0}$ on a submodule of $N$ such that:

- $f\left(\bigoplus_{k \geq 0} N_{k}\right) \subset \bigoplus_{k \geq 0} M_{k}$ and $f$ is a morphism of DG-modules of degree 0 with respect to both gradings;

$$
\begin{aligned}
& -g\left(M_{k}\right) \subset \bigoplus_{i \geq k} N_{i} \\
& -\phi\left(N_{k}\right) \subset \bigoplus_{i \geq k+1} N_{i} .
\end{aligned}
$$

Then the grading $\mathcal{G}$ is $\left(c^{\prime} \circ c\right)$-compatible.

Proof. Recall that

$$
\left(c^{\prime} \circ c\right):\left\{N, N^{\prime}, f^{\prime} f, g g^{\prime}, \phi+g \phi^{\prime} f\right\} .
$$


- Of course, $f^{\prime} f\left(N_{k}\right) \subset f^{\prime}\left(M_{k}\right)=0$ if $k \geq 1$, since $\mathcal{G}^{\prime}$ is $c^{\prime}$-compatible;

- Take now $\phi+g \phi^{\prime} f$. We must check that $g \phi^{\prime} f\left(N_{k}\right) \subset \bigoplus_{i \geq k+1} N_{i}$, but this is obvious due to the conditions imposed on $f$ and $g$ and the fact that $\mathcal{G}^{\prime}$ is $c^{\prime}$-compatible.

Notice that, given a contraction, one may construct lots of compatible gradings, so an important task is to determine which is a good one for our purposes, depending on the formula we want to reduce. The improvements achieved in each case will depend on the compatible grading considered.

\section{Application 1: The $A_{\infty}$-Structure of the Homology of $Q_{p}(u, 2 n)$}

Here we present an example of using compatible gradings to reduce complexity in the computation of some morphisms obtained by perturbation theory. We study the $A_{\infty}$-coalgebra structure of the homology $H_{*}\left(Q_{p}(u, 2 n) ; \mathbf{Z}\right)$ of a truncated polynomial algebra in the ring $\mathbf{Z}$.

An $A_{\infty}$-coalgebra is a DG-module $\left(M, \Delta_{1}\right)$ endowed with a family of morphisms of graded modules

$$
\Delta_{i}: M \rightarrow M^{\otimes i}
$$

of degree $i-2$ such that, for $n \geq 1$,

$$
\sum_{n=1}^{i} \sum_{k=0}^{i-n}(-1)^{n+k+n k}\left(1^{\otimes i-n-k} \otimes \Delta_{n} \otimes 1^{\otimes k}\right) \Delta_{i-n+1}=0 .
$$

To study the structure of the homology we need to consider the contraction

$$
c_{B Q}:\left\{\bar{B}\left(Q_{p}(u, 2 n)\right), E(v, 2 n+1) \otimes \Gamma(w, 2 n p+2), f_{B Q}, g_{B Q}, \phi_{B Q}\right\}
$$

From now on, $E(v, 2 n+1) \otimes \Gamma(w, 2 n p+2)$ will be denoted by $E \otimes \Gamma$ as well as $\bar{B}\left(Q_{p}(u, 2 n)\right)$ by $\bar{B}\left(Q_{p}\right)$, for short.

Then, an $A_{\infty}$-coalgebra structure is induced on $E \otimes \Gamma$, via the tensor trick (see [9, 12,11]) and the BPL (using as a perturbation datum the cosimplicial differential $\left.d_{c o s}\right)$. In fact, it is possible to construct a new contraction

$$
\bar{\Omega} \bar{B}\left(Q_{p}\right) \Rightarrow \widetilde{\Omega}(E \otimes \Gamma),
$$

where $\widetilde{\Omega}(E \otimes \Gamma)=\left(T s^{-1}(E \otimes \Gamma), \tilde{d}\right)$ and the morphism $\tilde{d}$, obtained by the perturbation process, induces an $A_{\infty}$-coalgebra structure $\left(\Delta_{1}, \Delta_{2}, \Delta_{3}, \ldots\right)$ on $(E \otimes \Gamma)$. Our aim is to use a compatible grading that allows a complexity reduction for the morphisms $\Delta_{i}$.

We denote an element of $\bar{B}\left(Q_{p}\right)$ of the form $\left[u^{r_{1}}|\ldots| u^{r_{m}}\right]$ by $\left[r_{1}|\ldots| r_{m}\right]$, where $0 \leq r_{i}<p$. 
The explicit morphisms of the contraction $c_{B Q}$ are described below:

- $f_{B Q}: \bar{B}\left(Q_{p}\right) \rightarrow E \otimes \Gamma$

$$
\begin{aligned}
& f_{B Q}\left[r_{1}\left|t_{1}\right| \ldots\left|r_{m}\right| t_{m}\right]=\left\{\prod_{k=1}^{m} \delta_{p, r_{k}+t_{k}}\right\} \gamma_{m}(w), \\
& f_{B Q}\left[l\left|r_{1}\right| t_{1}|\ldots| r_{m} \mid t_{m}\right]=\delta_{1, l}\left\{\prod_{k=1}^{m} \delta_{p, r_{k}+t_{k}}\right\} v \otimes \gamma_{m}(w),
\end{aligned}
$$

where the symbols $\delta_{i, j}$ are defined by: $\delta_{i, j}= \begin{cases}0 & \text { if } i \neq j \\ 1 & \text { if } i=j\end{cases}$

- $\left.g_{B Q}: E \otimes \Gamma \rightarrow \bar{B}\left(Q_{p}\right)\right)$ is defined over the algebra generators as follows:

$$
g_{B Q}(v)=[1], \quad g_{B Q}\left(\gamma_{k}(w)\right)=[1|p-1| \text { k times }|1| p-1] .
$$

- $\phi_{B Q}: \bar{B}\left(Q_{p}\right) \rightarrow \bar{B}\left(Q_{p}\right)$ is defined by:

$$
\begin{aligned}
& \phi_{B Q}[]=0 ; \\
& \phi_{B Q}[x]=-[1 \mid x-1] \quad 1<x<p \\
& \phi_{B Q}[x \mid y]=-[1|x-1| y] ; \\
& \phi_{B Q}([x \mid y] \mid z)=-[1|x-1| y]\left|z-\delta_{p, x+y}[1 \mid p-1]\right| \phi(z)
\end{aligned}
$$

where $z \in \bar{B}\left(Q_{p}\right)$ and $\mid$ denotes the juxtaposing product.

Let us consider the submodule $S$ of $\bar{B}\left(Q_{p}(u, 2 n)\right)$ generated by the elements $\left[a_{1}\left|a_{2}\right| \cdots \mid a_{r}\right]$ such that either $a_{2 i+1}=1$ for all $i$ or $a_{2 i}=1$ for all $i$.

We now construct a grading, $\mathcal{G}=\left\{S_{k}\right\}_{k \geq 0}$, over $S$ in the search of a $c_{B Q^{-}}$ compatible grading. Take the submodule $S_{k} \subset S$ described bellow:

$-S_{0}$ is generated by the elements of the form $[p-1|1| p-1 \mid 1 \cdots]$, of any simplicial dimension; $\left[a_{1}|1| a_{2} \mid 1 \cdots\right]$, with $a_{i}=p-1$ except for one of them, $a_{j}$, which satisfies $1 \leq a_{j} \leq p-2 ;[1|p-1| 1|p-1| 1 \cdots]$, of any simplicial dimension.

$-S_{k}$, for $k \geq 1$, is generated by the elements of

type I: $\left[1\left|a_{1}\right| \cdots|1| a_{r} \mid 1\right]$ or $\left[1\left|a_{1}\right| \cdots|1| a_{r}\right]$, where at least one component $a_{i} \neq 1$ and there are $k$ components (at even positions) satisfying $1 \leq a_{i} \leq p-2$

type II: $\left[a_{1}|1| a_{2}|\cdots| 1\right]$ or $\left[a_{1}|1| a_{2}|\cdots| a_{r}\right]$ where at least one component $a_{i} \neq$ 1 and there are $k+1$ components (at odd positions) satisfying $1 \leq a_{i} \leq p-2$;

type III: $[1|1| \cdots \mid 1]$ with simplicial dimension $2 k$ or $2 k+1$.

Then, it is easy to prove that $\mathcal{G}$ is $c_{B Q}$-compatible:

- Obviously, $f_{B Q}\left(S_{k}\right)=0$ for any $k \geq 1$; 
$-\phi\left(S_{i}\right) \subset \bigoplus_{j \geq i+1} S_{j}$. More specifically, it is easy to prove, by induction, that $\phi\left(S_{i}\right) \subset S_{i+1}$. Moreover, that $\phi($ elements of type II $)=($ elements of type I $)$ and that $\phi($ elements of type I $)=0=\phi($ elements of type III $)$.

The grading $\mathcal{G}$ on $S$ induces in a natural way, a grading over $T s^{-1}(S), T(\mathcal{G})$, as indicated in corollary 1 that is $T s^{-1} c_{B Q}$-compatible.

Moreover, the cosimplicial differential, $d_{c o s}$, which depends on the coproduct on $\bar{B}\left(Q_{p}\right)$ (see formula (1), is a perturbation datum for the latter contraction and satisfies

$$
d_{\text {cos }}\left(T(S)_{j}\right) \subset \oplus_{i \geq j-1} T(S)_{i} .
$$

In fact, it is easy to check that $d_{c o s}\left(T(S)_{j}\right) \subset T(S)_{j-1} \oplus T(S)_{j}$.

All these results lead to the following theorem.

Theorem 3. Let $T(\mathcal{G})$ be the grading naturally induced on $T s^{-1}(S)$ by corollary 1, being $\mathcal{G}$ the grading described above. Then $T(\mathcal{G})$ is $\left(T s^{-1} c_{B Q}, d_{\text {cos }}\right)$-compatible.

Now, we are in conditions of analyzing the consequences of having a $\left(T s^{-1} c_{B Q}\right.$, $d_{c o s}$ )-compatible grading in the computation of the $A_{\infty}$-coalgebra structure on $E \otimes \Gamma$.

The differential obtained by the BPL on $T s^{-1}(E \otimes \Gamma)$ is

$$
\tilde{d}=\sum_{i \geq 0}(-1)^{i} T(f) d_{c o s}\left(T(-\phi) d_{c o s}\right)^{i} T(g),
$$

and the morphisms $\Delta_{i}: E \otimes \Gamma \rightarrow(E \otimes \Gamma)^{\otimes i}$, providing an $A_{\infty}$-coalgebra structure on $E \otimes \Gamma$, are given by

$$
\Delta_{i}=(-1)^{[i / 2]} \uparrow^{\otimes i} f^{\otimes i}\left(d_{c o s} T(-\phi)\right)^{i-2} d_{c o s} g \downarrow
$$

Notice that $\operatorname{Im} g_{B Q} \subset S_{0}$ and that according to proposition 1 ,

$$
T(f)\left(d_{\cos } T(-\phi)\right)^{i}\left(\bigoplus_{k \geq 1}(T(S))_{k}\right)=0 \quad \text { for all } i \geq 0,
$$

so we can conclude that, since $T(-\phi)\left(T(S)_{k}\right) \subset T(S)_{k+1}$, we only must consider the summands of $d_{c o s}$ such that

$$
d_{c o s}\left(T(S)_{k}\right) \subset T(S)_{k-1} .
$$

This means that any time we apply $d_{c o s}$ to an element of cosimplicial dimension $m$, one can consider only one summand in formula 1 instead of $m$. That summand is the one that applies $\Delta_{\bar{B}}$ on the factor on which $\phi$ has just been applied. Moreover, since the output of $\phi$ is always a sum of elements of type I, we only need to consider the half of the summands of $\Delta_{\bar{B}}$ (those that decrease the degree by one). So, we could express the "reduced" formula of $\Delta_{i}$ by

$$
\bar{\Delta}_{i}=(-1)^{[i / 2]} \uparrow^{\otimes i} f^{\otimes i}\left(\bar{d}_{c o s} T(-\phi)\right)^{i-2} d_{c o s} g \downarrow
$$


where

$$
\bar{d}_{c o s}=1^{\otimes k} \otimes \downarrow^{\otimes 2} \bar{\Delta}_{\bar{B}} \uparrow \otimes 1^{\otimes n-k-1}
$$

and $k$ depends on the factor on which $\phi$ has been applied in $T(-\phi)$. Then,

$$
\bar{\Delta}_{\bar{B}}\left(\left[a_{1}|\cdots| a_{r}\right]\right)=\sum_{i=1}^{\lfloor r / 2\rfloor}\left[a_{1}|\cdots| a_{2 i-1}\right] \otimes\left[a_{2 i}|\cdots| a_{r}\right] .
$$

Finally, we expose some examples in order to illustrate the improvements achieved in complexity. The calculations have been made (up to signs) using MATHEMATICA 4.0, in which we have implemented the formulas of $\Delta_{i}$ and $\bar{\Delta}_{i}$, in a Pentium IV $1,6 \mathrm{GHz} 512 \mathrm{MB}$ RAM.

Firstly, we will express the time used in the computation of $\Delta_{3}\left(\gamma_{k}(w)\right)$ for different values of $k$ in the case $p=3$.

\begin{tabular}{c|cccc} 
Time used (Seconds) & $k=5$ & $k=10$ & $k=20$ & $k=30$ \\
\hline$\Delta_{3}$ & 0.15 & 1.072 & 50.282 & 882,959 \\
$\bar{\Delta}_{3}$ & 0.07 & 0.391 & 5.859 & 77.392
\end{tabular}

The following table shows the time used in computing $\Delta_{i}\left(\gamma_{5}(w)\right)$ versus $\bar{\Delta}_{i}\left(\gamma_{5}(w)\right)$ for different values of $i$ and $p$.

\begin{tabular}{c|cccc} 
Time used (seconds) & $i=3=p$ & $i=4=p$ & $i=5=p$ & $i=6=p$ \\
\hline$\Delta_{i}$ & 0.15 & 1.081 & 16.373 & 226.456 \\
$\bar{\Delta}_{i}$ & 0.07 & 0.311 & 2.043 & 16.213
\end{tabular}

In the last table we expose the number of summands at different stages of the computation of $\Delta_{6}\left(\gamma_{5}(w)\right)$ as well as $\bar{\Delta}_{6}\left(\gamma_{5}(w)\right)$ in the case $p=6$.

\begin{tabular}{c|cccc} 
Number of summands & $i=1$ & $i=2$ & $i=3$ & $i=4$ \\
\hline$\left(d_{\text {cos }} T(-\phi)\right)^{i} d_{\cos } g\left(\left\langle\gamma_{5}(w)\right\rangle\right)$ & 135 & 945 & 4410 & 15876 \\
$\left(\bar{d}_{\text {cos }} T(-\phi)\right)^{i} d_{\text {cos }} g\left(\left\langle\gamma_{5}(w)\right\rangle\right)$ & 55 & 315 & 1274 & 4116
\end{tabular}

\section{Application 2: On the 1-Homological Model of a Commutative Connected DG-Algebra}

In 44 and 14 a strategy was developed, called "inversion theory", with the goal of improving the computation of some formulas (obtained by the BPL) involved 
in the 1-homological model of a commutative DGA-algebra (see [1]). In this section, we will see that this theory fits perfectly in the framework developed before, so we will briefly review the concepts given in the inversion theory under this new viewpoint to realize that, in fact, it can be considered as an application of the general methodology of this paper.

Every commutative DGA-algebra $A$ is quasi-isomorphic (there is a homomorphism that induces an isomorphism in homology) to a twisted tensor product (TTP) of exterior and polynomial algebras, that is, a tensor product $\otimes_{i \in I} A_{i}$, each $A_{i}$ being an exterior or a polynomial algebra, endowed with an additional differential structure $\rho$ (see [3] ).

Here we will restrict to the case of commutative connected DG-algebras, in order to deal with simpler formulas.

Take, then, a TTP of exterior and polynomial algebras $A=\left(\otimes_{i \in I} A_{i}, \rho\right)$. In [1] a process is described consisting in composition and tensor product of contractions followed by the application of the BPL in order to obtain a new contraction called a 1-homological model for $A$ :

$$
c_{\delta}:\left\{\left(\bar{B}\left(\otimes_{i \in I} A_{i}\right), \delta\right),\left(\otimes_{i \in I} h B A_{i}, d_{\delta}\right), f_{\delta}, g_{\delta}, \phi_{\delta}\right\}
$$

where $\delta$ is a perturbation on $\bar{B}\left(\otimes_{i \in I} A_{i}\right)$ induced by $\rho$ and $h B A_{i}$ is an exterior or a divided polynomial algebra, depending on whether $A_{i}$ was a polynomial or an exterior algebra. The search of a $(c, \delta)$-compatible grading on $\bar{B}\left(\otimes_{i \in I} A_{i}\right)$ is motivated by the high computational cost of $d_{\delta}$, which is of exponential nature, since shuffles are involved in the formulas.

The construction of $c_{\delta}$ makes use of three basic contractions:

- The contraction given in [7]:

$$
c_{B P}:\left\{\bar{B}(P(v, 2 n)), E(s(v), 2 n+1), f_{B P}, g_{B P}, \phi_{B P}\right\} .
$$

If an element $\left[v^{r_{1}}|\cdots| v^{r_{m}}\right]$ is denoted by $\left[r_{1}|\cdots| r_{m}\right]$ for short, then,

$$
\begin{gathered}
f_{B P}([r])=\left\{\begin{array}{ll}
0 & \text { if } r \neq 1 \\
s(v) & \text { if } r=1
\end{array} ; f_{B P}\left(\left[r_{1}|\cdots| r_{m}\right]\right)=0\right. \\
g_{B P}(s v)=[1] ; \quad \phi_{B P}\left(\left[r_{1}|\cdots| r_{m}\right]\right)=\left[1\left|r_{1}-1\right| \cdots \mid r_{m}\right] .
\end{gathered}
$$

- The isomorphism of DG-algebras (also described in [7]):

$$
c_{B E}:\left\{\bar{B}(E(u, 2 n+1)), \Gamma(s(u), 2 n+2), f_{B E}, g_{B E}, 0\right\},
$$

where

$$
f_{B E}\left(\left[\left.u\right|^{m \text { times }} \mid u\right]\right)=\gamma_{m}(s(u)) ; g_{B E}\left(\gamma_{m}(s(u))\right)=\left[\left.u\right|^{m \text { times }} \mid u\right] .
$$

- Let $A$ and $A^{\prime}$ be two commutative connected $D G$-algebras. There is a contraction $c_{B \otimes}:\left\{\bar{B}\left(A \otimes A^{\prime}\right), \bar{B}(A) \otimes \bar{B}\left(A^{\prime}\right), f_{B \otimes}, g_{B \otimes}, \phi_{B \otimes}\right\}$ (see [7]), whose formulas (for the connected case) are recalled here: 
- $f_{B \otimes}$ is null except for the case $f_{B \otimes}\left[a_{1} \otimes 1|\cdots| a_{i} \otimes 1\left|1 \otimes a_{i+1}^{\prime}\right| \cdots \mid 1 \otimes a_{n}^{\prime}\right]=\left[a_{1}|\cdots| a_{i}\right] \otimes\left[a_{i+1}^{\prime}|\cdots| a_{n}^{\prime}\right]$.

- $g_{B \otimes}\left(\left[a_{1}|\cdots| a_{n}\right] \otimes\left[a_{1}^{\prime}|\cdots| a_{m}^{\prime}\right]\right)=\left[a_{1}|\cdots| a_{n}\right] \star\left[a_{1}^{\prime}|\cdots| a_{m}^{\prime}\right]$.

- $\phi_{\bar{B} \otimes}\left(\left[a_{1} \otimes a_{1}^{\prime}|\cdots| a_{n-q} \otimes a_{n-q}^{\prime}\left|a_{n-q+1}^{\prime}\right| \cdots \mid a_{n}^{\prime}\right]\right)$

$$
\begin{aligned}
=\sum_{p=0}^{n-q-1} \sum_{\pi} \pm\left[a_{1} \otimes a_{1}^{\prime}|\cdots| a_{\bar{n}-1} \otimes a_{\bar{n}-1}^{\prime} \mid\right. \\
\left.\left(a_{\bar{n}}^{\prime} *_{A^{\prime}} \cdots *_{A^{\prime}} a_{n-q}^{\prime}\right)\left|c_{\pi(0)}\right| \cdots \mid c_{\pi(p+q)}\right],
\end{aligned}
$$

where $\pi$ runs over the $\{(p+1, q)$-shuffles $\}, \bar{n}=n-p-q$ and

$$
\left(c_{0}, \ldots, c_{p+q}\right)=\left(a_{\bar{n}}, \ldots, a_{n-q}, a_{n-q+1}^{\prime}, \ldots a_{n}^{\prime}\right) .
$$

Notice that $g_{B \otimes}$ and $\phi_{B \otimes}$ works in exponential time due to the shuffles involved.

So, for a commutative connected DG-algebra $\left(A \otimes A^{\prime}, \rho\right)$, being $A$ and $A^{\prime}$ an exterior or polynomial algebra, one can construct, by composition and tensor product of the previous contractions, the contraction $c=\left(c_{B A} \otimes c_{B A^{\prime}}\right) \circ c_{B \otimes}$,

$$
(f, g, \phi): \bar{B}\left(A \otimes A^{\prime}\right) \Rightarrow \bar{B}(A) \otimes \bar{B}\left(A^{\prime}\right) \Rightarrow h B A \otimes h B A^{\prime}
$$

where $h B A$ as well as $h B A^{\prime}$ are either a polynomial or a divided polynomial algebra depending on whether $A$ and $A^{\prime}$ were, respectively, an exterior or a polynomial algebra. The morphisms are given by

$$
\begin{aligned}
& f=\left(f_{B A} \otimes f_{B A^{\prime}}\right) f_{B \otimes} \\
& g=g_{B \otimes}\left(g_{B A} \otimes g_{B A^{\prime}}\right) \\
& \phi=\phi_{B \otimes}+g_{B \otimes}\left(\phi_{B A} \otimes g_{B A^{\prime}} f_{B A^{\prime}}+1_{B A} \otimes \phi_{B A^{\prime}}\right) f_{B \otimes}
\end{aligned}
$$

Take the contraction $c_{B P}$. Consider the grading $\mathcal{G}_{B P}=\left\{G_{k}^{B P}\right\}_{k \geq 0}$ by which $G_{k}^{B P}$ is the submodule generated by the elements of the form $\left[r_{1}|\cdots| r_{k+1}\right]$ (that is, those of simplicial degree $k+1$ ). It is easy to check that $\mathcal{G}_{B P}$ is a $c_{B P}-$ compatible grading.

Consider, now, the contraction $c_{B E}$. The grading $\mathcal{G}_{B E}=\left\{G_{k}^{B E}\right\}_{k \geq 0}$ with $G_{0}^{B E}=\bar{B}(E(u, 2 n+1))$ and $G_{k}^{B E}=0$ for $k \geq 0$ is, trivially, a $c_{B E}$-compatible grading.

Then, $\mathcal{G}_{B A} \otimes \mathcal{G}_{B A^{\prime}}$ is a $c_{B A} \otimes c_{B A^{\prime}}$-compatible grading, by theorem 1

Then, we must look for a grading on $\bar{B}\left(A \otimes A^{\prime}\right)$ (or a submodule of it) such that the conditions stated in theorem 2 are satisfied, in order to have a $c$-compatible grading.

Take the notation $\mathcal{G}_{B A} \otimes \mathcal{G}_{B A^{\prime}}=\left\{G_{k}^{\otimes B}\right\}_{k \geq 0}$, meanwhile the desired grading on $\bar{B}\left(A \otimes A^{\prime}\right)$ will be denoted by $\mathcal{G}_{B \otimes}=\left\{G_{k}^{B \otimes}\right\}_{k \geq 0}$.

Let us consider $G_{k}^{B \otimes}$ as the submodule of $\bar{B}\left(A \otimes A^{\prime}\right)$ generated by the elements with $k$ inversions as defined in [14: Let $\bar{a}=\left[a_{1} \otimes a_{1}^{\prime}\left|a_{2} \otimes a_{2}^{\prime}\right| \cdots \mid a_{n} \otimes a_{n}^{\prime}\right]$ be an homogeneous element of $\bar{B}\left(A \otimes A^{\prime}\right)$. The component $a_{i} \otimes a_{i}^{\prime}$ is said to be an inversion if one of the the following cases occurs: 
$-a_{i}=1$ and there exists an index $j>i$ with $a_{j} \in \bar{A}$ (we say that $a_{i}$ is a Q-inversion);

- $A^{\prime}$ is a polynomial algebra and $a_{i-1}=1=a_{i}=\cdots=a_{n}$ (we say that $a_{i}$ is a $p$-inversion).

- $A$ is a polynomial algebra, $a_{i} \in \bar{A}$ and there exists an index $j>i$ such that $a_{j} \in \bar{A}$ (we say that $a_{i}$ is a $p 1$-inversion).

This grading verifies the conditions in theorem 2 .

- $f_{B \otimes}$ is a morphism of degree 0 with respect to the gradings $\mathcal{G}_{B \otimes}$ and $\mathcal{G}_{B A} \otimes$ $\mathcal{G}_{B A^{\prime}}$ : This is obvious, since $f_{B \otimes}(\bar{a}) \neq 0$, only for the elements of the form $\bar{a}=\left[a_{1}|\cdots| a_{j}\left|a_{1}^{\prime}\right| \cdots \mid a_{k}^{\prime}\right]$, with $a_{i} \in \bar{A}$ and $a_{i}^{\prime} \in \bar{A}^{\prime}$, which has the same degree in $\mathcal{G}_{B \otimes}$ than $\left[a_{1}|\cdots| a_{j}\right] \otimes\left[a_{1}^{\prime}|\cdots| a_{k}^{\prime}\right]$ in $\mathcal{G}_{B A} \otimes \mathcal{G}_{B A^{\prime}}$.

$-g_{B \otimes}\left(G_{k}^{\otimes B}\right) \subset \bigoplus_{i \geq k} G_{i}^{B \otimes}$ : this is easy to see since $g_{B \otimes}$ makes the shuffle product.

- $\phi\left(G_{k}^{B \otimes}\right) \subset \bigoplus_{i \geq k+1} G_{i}^{B \otimes}:$

Recall that $\phi=\phi_{B \otimes}+g_{B \otimes}\left(\phi_{B A} \otimes g_{B A^{\prime}} f_{B A^{\prime}}+1_{B A} \otimes \phi_{B A^{\prime}}\right) f_{B \otimes}$ and that either $\phi_{B \otimes}$ or $\phi_{B P}$ always produce at least one more inversion [4, 14].

So, $\mathcal{G}_{B \otimes}$ is a $c$-compatible grading on $B\left(A \otimes A^{\prime}\right)$.

Now, take a perturbation $\rho$ of the tensor product $A \otimes A^{\prime}$, then $\rho$ produces a perturbation

$$
\delta=-\sum_{i=0}^{n-1} 1^{\otimes i} \otimes \uparrow \rho \downarrow \otimes 1^{\otimes n-i-1}
$$

on $\bar{B}\left(A \otimes A^{\prime}\right)$. Then we can state the following result.

Theorem 4. The grading $\mathcal{G}_{B \otimes}$ on $B\left(A \otimes A^{\prime}\right)$ described above is $(c, \delta)$-compatible.

Proof. We must check that $\delta\left(G_{k}^{B \otimes}\right) \subset \bigoplus_{i \geq k-1} G_{i}^{B \otimes}$, for any $k \geq 1$. The key of the proof lays in the fact that each summand of $\delta$ acts only on one component $a_{i} \otimes a_{i}^{\prime}$ of the element $\left[a_{1} \otimes a_{1}^{\prime}|\cdots| a_{n} \otimes a_{n}^{\prime}\right]$. So, at most, only one inversion can disappear. On the contrary, if $\rho$ is applied to a component which is not implied in any inversion, the summand obtained could have an amount of inversions greater or equal to the original one.

We then have a $(c, \delta)$-compatible grading, what means that

$$
f(\delta \phi)^{i}\left(\bigoplus_{k \geq 1} G_{k}^{B \otimes}\right)=0 \quad \text { for all } i \geq 0 .
$$

This way, we can conclude that, in order to compute $d_{\delta}$, whose formula is

$$
d_{\delta}=\sum_{i \geq 0}(-1)^{i} f \delta(\phi \delta)^{i} g,
$$

we can ignore those summands of $\phi$ that increase more than by one the degree of the element. This conclusion leads to the following result already proved by inversion theory. 
Corollary 3. [4, 14] The formula for $\phi$, involved in the perturbed differential, $d_{\delta}$, of the 1-homological model of a commutative connected DG-algebra $(A \otimes$ $\left.A^{\prime}, \rho\right)$ can be reduced to the following one:

$$
\phi=\bar{\phi}_{B \otimes}+\bar{g}_{B \otimes}\left(\phi_{B A} \otimes g_{B A^{\prime}} f_{B A^{\prime}}+1 \otimes \phi_{B A^{\prime}}\right) f_{B \otimes},
$$

where

$$
\begin{gathered}
-\bar{\phi}_{B \otimes}\left(\left[a_{1} \otimes a_{1}^{\prime}|\cdots| a_{n} \otimes a_{n}^{\prime}\right]\right) \\
=\sum_{0 \leq p \leq n-q-1 \leq n-1} \pm\left[a_{1} \otimes a_{1}^{\prime}|\cdots| a_{\bar{n}-1} \otimes a_{\bar{n}-1}^{\prime} \mid\left(a_{\bar{n}}^{\prime} *_{A^{\prime}} \cdots *_{A^{\prime}} a_{n-q}^{\prime}\right)\right. \\
\left.\left|a_{\bar{n}}\right| \cdots\left|a_{n-q}\right| a_{n-q+1}^{\prime}|\cdots| a_{n}^{\prime}\right] . \\
-\bar{g}_{B \otimes}\left(\left[a_{1}|\cdots| a_{n}\right] \otimes\left[a_{1}^{\prime}|\cdots| a_{m}^{\prime}\right]\right)=\left[a_{1}|\cdots| a_{n}\left|a_{1}^{\prime}\right| \cdots \mid a_{m}^{\prime}\right] .
\end{gathered}
$$

This way, the formula of $\phi_{B \otimes}$ comes out to be of quadratic instead of exponential order. On the other hand, the formula for $g_{B \otimes}$ is reduced to 1 summand in contrast with the original $\left(\begin{array}{c}m+n \\ n\end{array}\right)$.

These results can be extended, recursively, to the general case of a commutative connected DG-algebra factored as a twisted tensor product $\left(\otimes_{i=1}^{n} A_{i}, \rho\right)$, being $A_{i}$ an exterior or polynomial algebra, $i=1,2, \ldots, n$ and $\rho$ a differential, such that $\rho\left(A_{k}\right) \subset \otimes_{i=1}^{k} A_{i}$.

\section{References}

1. Álvarez, V., Armario, A., Real, P., Silva, B.: HPT and computability of Hochschild and cyclic homologies of commutative DGA-algebras. In: Conference on Secondary Calculus and Cohomological Physics, Moscow. EMIS Electronic Proceedings, http://www.emis.de/proceedings, 1997

2. Brown, R.: The twisted Eilenberg-Zilber theorem, Celebrazioni Archimedae del Secolo XX, Simposio di Topologia (1967), 34-37

3. Burghelea, D., Vigué Poirrier, M.: Cyclic homology of commutative algebras I, Lecture Notes in Mathematics, Algebraic Topology Rational Homotopy 1318, 51$72(1986)$

4. C.H.A.T.A. group. Computing "small" 1-homological models for Commutative Differential Graded Algebras. In: Proc. Third Workshop on Computer Algebra in Scientific Computing, Springer-Verlag, Berlin, Heidelberg (2000) 87-100

5. Cartan, H., Eilenberg, S.: Homological Algebra, Princeton Univ. Press, 1956

6. Eilenberg, S., MacLane, S.: On the groups $H(\pi, n)$, I, Annals of Math. 58 (1953), 55-106

7. Eilenberg, S., MacLane, S.: On the groups $H(\pi, n)$, II, Annals of Math. 60 (1954), 49-139

8. Gugenheim, V.K.A.M.: On the chain complex of a fibration, Illinois J. Math. 3 (1972), 398-414

9. Gugenheim, V.K.A.M.: On Chen's iterated integrals, Illinois J. Math. (1977), $703-715$ 
10. Gugenheim, V.K.A.M., Lambe, L.: Perturbation theory in Differential Homological Algebra, I, Illinois J. Math. 33 (1989), 56-582

11. Gugenheim, V.K.A.M., Lambe, L., Stasheff, J.: Perturbation theory in differential homological algebra, II, Illinois J. Math. 35(3) (1991), 357-373

12. Gugenheim, V. K. A. M., Stasheff, J.: On perturbations and $A_{\infty}$-structures, Bull. Soc. Math. Belg. 38 (1986), 237-246

13. Huebschmann, J., Kadeishvili, T.: Small models for chain algebras, Math. Zeit. 207 (1991), 245-280

14. Jiménez, M.J., Real, P.: "Coalgebra" structures on 1-homological models for commutative differential graded algebras. In: Proc. Fourth Workshop on Computer Algebra in Scientific Computing, Springer-Verlag, Berlin, Heidelberg (2001) 347361

15. Lambe, L.A., Stasheff, J.: Applications of perturbation theory to iterated fibrations, Manuscripta Math. 58 (1987), 363-376

16. MacLane, S.: Homology, Classics in Mathematics, Springer-Verlag, Berlin, 1995. Reprint of the 1975 edition

17. Real, P.: Homological perturbation theory and associativity, Homology, Homotopy and Applications 2(5) (2000), 51-88

18. Shih, W.: Homologie des espaces fibrés, Inst. Hautes Etudes Sci. 13 (1962), 93-176

19. Weibel, C.A.: An introduction to Homological Algebra, Cambridge studies in advanced mathematics 38, Cambridge University Press, 1994 